\title{
EXPERIENCIA DE LA APLICACION DEL PROTOCOLO DE EVALUACIÓN DE BUENAS PRÁCTICAS EN PARENTALIDAD POSITIVA EN EL PROGRAMA DE ACOGIMIENTO RESIDENCIAL DE MENORES GESTIONADO POR LA ENTIDAD MENSAJEROS DE LA PAZ- CANARIAS
}

\author{
Sabrina María Henríquez Jiménez \\ sabrina@mensajerosdelapazcanarias.com \\ Omaira Teresa Segura Robaina
}

Fecha de Recepción: 19 Enero 2019

Fecha de Admisión: 30 Abril 2019

\section{RESUMEN}

Teniendo como fin alcanzar una mejora del servicio de atención a las familias en el programa de Acogimiento residencial de menores gestionado por la entidad Mensajeros de la Paz Canarias, la cual gestiona en la actualidad 12 hogares de protección de menores en la isla de Gran Canaria, se ha realizado una evaluación a través del protocolo de Buenas Prácticas desde el enfoque de la Parentalidad Positiva considerando que el servicio requiere intervenir con las familias desde este enfoque como forma de profesionalizar el servicio y a su vez ofrecer desde la entidad una atención de calidad que permita crear y mantener una relación de ayuda favorable y lograr la reunificación familiar cuando el Plan de Caso así lo requiera.

Tras la aplicación del protocolo a través de un proceso riguroso con la participación de los profesionales que intervienen tanto de manera directa como indirecta en los casos y siguiendo las fases de aplicación tal y como recomienda la Guía de Buenas Prácticas en Parentalidad Positiva se han obtenido resultados relativos al servicio que requieren ser mejorados. Se ha elaborado un plan de mejora que en la actualidad la entidad se encuentra en el proceso de implementación, no obstante, estos cambios ya están produciendo mejoras tanto en las respuestas a las intervenciones de las propias familias como en el trabajo diario de los profesionales poniendo en marcha una metodología de trabajo rigurosa, consensuada y conjunta por parte de todo el equipo.

Palabras clave: parentalidad positiva; guía; plan de caso; acogimiento residencial; atención de calidad

\section{ABSTRACT \\ The experience in the application of the protocol of the evaluation about the good practices in the service of attention to the families from the approach of the positive parenting. With the aim of achieving an improvement in the service for families in the residential foster care program managed by the entity Mensajeros de la Paz Canarias, which currently manages 12 child protection homes on}




\section{EXPERIENCIA DE LA APLICACION DEL PROTOCOLO DE EVALUACIÓN DE BUENAS PRÁCTICAS EN PARENTALIDAD POSITIVA EN EL PROGRAMA DE ACOGIMIENTO RESIDENCIAL DE MENORES GESTIONADO POR LA ENTIDAD MENSAJEROS DE LA PAZ- CANARIAS}

the island of Gran Canaria, an evaluation has been carried out through the Good Practices protocol from the Positive Parenting approach, considering that the service requires intervention with families from this approach as a way to professionalize the service and, and at the same time offer quality care from the entity that allows creating and maintaining a favorable support relationship and achieve family reunification when the Case Plan so requires.

After the application of the protocol through a rigorous process with the participation of the professionals who intervene both directly and indirectly in the cases and following the application phases as recommended by the Good Practices Guide in Positive Parenting, results have been obtained relating to the service that needs to be improved. An improvement plan has been drawn up that the entity is currently in the implementation process, however, these changes are already producing improvements both in the responses to the interventions of the families themselves and in the daily work of the professionals. A rigorous, consensual and joint work methodology on the part of the entire team.

Keywords: positive parenting; guide; case plan; residential care; quality care

\section{INTRODUCCIÓN}

Antes de ahondar dentro del marco teórico de la evaluación de buenas practicas profesionales resulta importante definir que implica el concepto de "buena práctica". Tomando como referencia la Guía de Buenas Prácticas en parentalidad positiva se refiere a "toda experiencia, guiada por principios, objetivos y procedimientos apropiados, que ha arrojado resultados positivos, demostrando su eficacia y utilidad en un contexto concreto, pues bien, cuando nos referimos a las buenas prácticas profesionales este concepto puede referirse a un continuo de experiencias que van desde el sentido común 0 el uso tradicional de determinadas formas de hacer 0 actuar, hasta la práctica profesional basada en evidencias científicas." Así, se distinguen tres niveles de buenas prácticas:

-Nivel 1. Práctica profesional individualizada basada en el conocimiento práctico recibido, en la propia experiencia del profesional 0 en la observación directa de las prácticas de otros profesionales en su entorno.

-Nivel 2. Práctica profesional consensuada. Consiste en el esfuerzo de transmisión de las prácticas de unos profesionales a otros hasta crear unas condiciones suficientes para llegar al consenso entre todos.

-Nivel 3. Práctica profesional basada en evidencias la cual requiere trasladar los resultados de la investigación en aplicaciones prácticas, hacer llegar dichos resultados de manera resumida y asequible a los y las profesionales, difundirlos y divulgarlos para que se constituyan en una práctica informada.

\section{EVALUACIÓN DE BUENAS PRÁCTICAS EN EL SERVICIO DE ATENCIÓN DE APOYOS A LA FAMILIA DESDE EL ENFOQUE DE LA PARENTALIDAD POSITIVA}

De acuerdo con la definición acuñada en la Recomendación Rec (2006) 19 del Comité de Ministros del Consejo de Europa "ejercicio de la parentalidad positiva se refiere al comportamiento de los padres fundamentado en el interés superior del niño, que cuida, desarrolla sus capacidades, no es violento y ofrece reconocimiento y orientación que incluyen el establecimiento de límites que permitan el pleno desarrollo del niño".

Desde la práctica profesional se entiende el enfoque de parentalidad positiva con las familias teniendo en cuenta que el ejercicio de la parentalidad positiva no se ejerce en un espacio vacío, sino que requiere de aliados y de apoyos materiales, de información y consejo, de acompañamiento, de comprensión y de carácter formativo para mejorar su ejercicio. Todos estos apoyos, que proporcio- 
nan personas e instituciones, favorecen el bienestar físico y psicológico de los miembros de la familia, reduciendo el impacto de los acontecimientos estresantes y promoviendo un sentimiento de identidad, de autoestima y de integración a la sociedad.

Para evaluar de manera rigurosa el servicio que se presta a las familias desde este enfoque se ha diseñado la Guía de Buenas Prácticas de Parentalidad Positiva que parte de la experiencia científica, esto es de conocimientos cientifico-aplicados y los conocimientos profesionales que han surgido como fruto del consenso durante la práctica, que se adaptan a personas, familias y comunidades concretas, y que se aplican dentro del marco deontológico de actuación profesional y del contexto legal, organizativo y comunitario que les da sentido y legitimidad. Esta Guía tiene como objetivo general introducir el enfoque de parentalidad positiva en los servicios de apoyo a las familias para potenciar los procesos de innovación y de mejora de la calidad mediante cambios en las culturas organizativas y en las prácticas profesionales. constituye una herramienta inestimable para evaluar las propias condiciones del servicio, así como la idoneidad y pertinencia de las actuaciones profesionales ya estén enmarcadas o no en programas.

Es importante señalar que como instrumento de evaluación la Guía contiene un Protocolo de Buenas Practicas que recoge aquellos aspectos que caracterizan el quehacer profesional y el servicio de apoyo a las familias que son relevantes desde el enfoque de la parentalidad positiva y que pueden ser objeto de mejora del servicio que se oferta.

El protocolo se articula de forma inclusiva en tres niveles, situados de mayor a menor generalidad: Ámbitos de contenido, Buenas prácticas e indicadores.

Respecto al primer nivel, las posibilidades de mejora de la práctica profesional y del servicio se exploran en tres Ámbitos de contenido claramente interconectados:

Características de los servicios de apoyo a las familias y cultura organizacional.

Proceso del trabajo profesional con las familias.

Uso de programas basados en evidencias en apoyo a las familias.

Estos tres ámbitos constituyen ejes importantes del contenido del protocolo que sirven para organizar las mejoras en torno a estos. Dicho protocolo evalúa competencias y prácticas profesionales del servicio en general con el fin de encontrar formas de apoyar el progreso del servicio y de sus profesionales.

\section{CARACTERÍSTICAS Y SERVICIOS DEL PROGRAMA DE ACOGIMIENTO RESIDENCIAL CON PLAN DE CASO DE REUNIFICACIÓN FAMILIAR}

El programa de acogimiento residencial de menores es una medida de amparo del sistema de protección que persigue unos objetivos concretos, prioritariamente enfocados a la reunificación familiar y con una temporalidad limitada, por ello, toda intervención debe estar orientada a la consecución del objetivo de la reintegración familiar, revisable en función de cómo evolucione el menor, su familia y sus necesidades, salvo que dicha reunificación resultara imposible para interés superior del menor.

El Plan de Caso siempre debe estar orientado hacia la familia de origen de los/as menores así como hacia el menor, la menor 0 adolescente cuando sea viable y no existan indicadores que desaconsejen esta línea de intervención.

La aplicación de este programa tiene como finalidad fomentar en todos los miembros de la familia la adquisición de habilidades básicas y hábitos de conductas necesarios para superar la situación de vulnerabilidad llevando a cabo evaluaciones continuas de la problemática familiar que permita ofrecer un pronóstico positivo. 


\section{EXPERIENCIA DE LA APLICACION DEL PROTOCOLO DE EVALUACIÓN DE BUENAS PRÁCTICAS EN PARENTALIDAD POSITIVA EN EL PROGRAMA DE ACOGIMIENTO RESIDENCIAL DE MENORES GESTIONADO POR LA ENTIDAD MENSAJEROS DE LA PAZ- CANARIAS}

\section{Objetivo General.}

La intervención familiar se basará en un trabajo que consiga que el niño/a, o adolescente retorne a la familia en el plazo de tiempo más breve posible, garantizando la satisfacción de sus necesidades básicas y debe centrarse en la evaluación de las necesidades familiares y no sólo en los incidentes que hayan podido producirse.

\section{Objetivos Específicos.}

Los objetivos por tanto de esta propuesta, siempre en colaboración con el resto de los profesionales que intervengan en el caso, siendo especialmente importante la adecuada coordinación con los Equipos que desarrollan el programa de intervención familiar son:

El aumento del bienestar familiar, que es el objetivo de la intervención que permitirá erradicar el problema.

Conseguir que el niño, niña o adolescente retorne a la familia en el plazo de tiempo más breve posible, garantizando la satisfacción de sus necesidades básicas.

Capacitar a los padres, madres, tutores o guardadores para ejercer adecuadamente el rol parental.

Eliminar o reducir el riesgo para que la desprotección no vuelva a aparecer en el fututo.

Reparar las secuelas que la desprotección ha provocado en el niño, niña 0 adolescente.

\section{APLICACIÓN DEL PROTOCOLO}

La aplicación del Protocolo de Buenas Prácticas en Parentalidad Positiva se ha implementado siguiendo las recomendaciones expuestas en la Guía en la medida de lo posible. Se ha contado con el respaldo de la entidad y responsables de la Asociación Mensajeros de la Paz- Canarias.

Su aplicación ha involucrado a los/as profesionales relacionados con el servicio basándose en sus conocimientos y experiencias. Aunque no se ha involucrado de manera directa a las familias que hacen uso del servicio, se ha tenido especial importancia a lo expresado por los usuarios/as durante el proceso de intervención y teniéndose presente sus opiniones a la hora de aplicar los indicadores.

Se ha involucrado a otros servicios, en este caso ha participado en la parte 1 y 2 del protocolo un técnico de la sección de acogimiento de menores del Cabildo de Gran Canaria el cual comparte responsabilidad y coordinación directa con los profesionales del servicio. Este profesional cuenta con formación en la aplicación de la Guía habiendo participado en la mesa de trabajo para la elaboración de ésta.

El grupo ha estado formado por 5 profesionales, además de por el técnico mencionado anteriormente por la coordinadora del servicio y programas de la entidad Mensajeros de la Paz Canarias, dos directoras (trabajadoras sociales) de hogares de protección de la red insular, una trabajadora social novel (7 meses interviniendo en el programa).

\section{PROCESO DE APLICACIÓN}

El protocolo consta de 4 fases, en este apartado se desarrollan las 2 primeras que están relacionadas con las acciones realizadas previa a la formación del grupo, su creación y la respuesta del grupo dinamizador a los indicadores:

Fase 1. Trabajo con el protocolo: Explicar la aplicación de la Guía de Buenas Prácticas en Parentalidad Positiva, explicar el enfoque de parentalidad positiva y el objetivo de la aplicación del protocolo como forma de evaluación del servicio con el fin de planificar mejoras en la entidad. Se ha facilitado la Guía y el folleto de buenas prácticas profesionales para el apoyo a la parentalidad positiva a los participantes para que se familiaricen con los conceptos, términos, así como con los 
ámbitos e indicadores antes de la formación del grupo y la aplicación de éste. Los participantes han mostrado motivación ante la iniciativa, considerándolo como propuesta positiva para la profesionalización del servicio. Una vez realizada la difusión se han resuelto dudas que han presentado previo a la formación del grupo y aplicación del protocolo.

Fase 2. Analizar el servicio y actuación profesional: El protocolo se fue cumplimentando entre todos previamente en formato papel puesto que se generaron ciertos debates basados en la experiencia profesional a la cual el grupo debía llegar a un consenso en ciertos indicadores. Respecto a la Parte 1 se generó un debate ya que ciertos indicadores no procedían responder debido a la particularidad del servicio puesto que los casos que llegan al programa de acogimiento residencial llegan derivados de otros recursos de la red (Fiscalía de menores, C.A.I, Dirección General de la protección de la Infancia y la Familia) y no a petición de los familiares por lo que el acceso al servicio llega prácticamente impuesto a los usuarios/as. Lo mismo ocurre con la difusión del servicio que no se dirige a la población en general ya que los usuarios/as de este son los/as familiares presentes en la intervención familiar del menor declarado en situación de desamparo.

Finalmente se incorporan los resultados obtenidos directamente a la aplicación para la obtención del informe final. Cabe destacar que de la interacción del grupo se crea un espacio de intercambio donde se analiza la práctica profesional, los éxitos y dificultades obtenidos de la experiencia profesional. Se exponen intervenciones y ejemplos de casos concretos trabajados en el servicio, la forma en la que se intervino, cómo se alcanzó el vínculo y la calidad de la relación de ayuda profesional con las familias y los resultados obtenidos

\section{RESULTADO: INFORME FINAL}

PARTE 1. Buenas prácticas en el servicio desde el enfoque de la parentalidad positiva.

Figura 1. Resultados del protocolo de Buenas Prácticas en parentalidad positiva. FEMP.

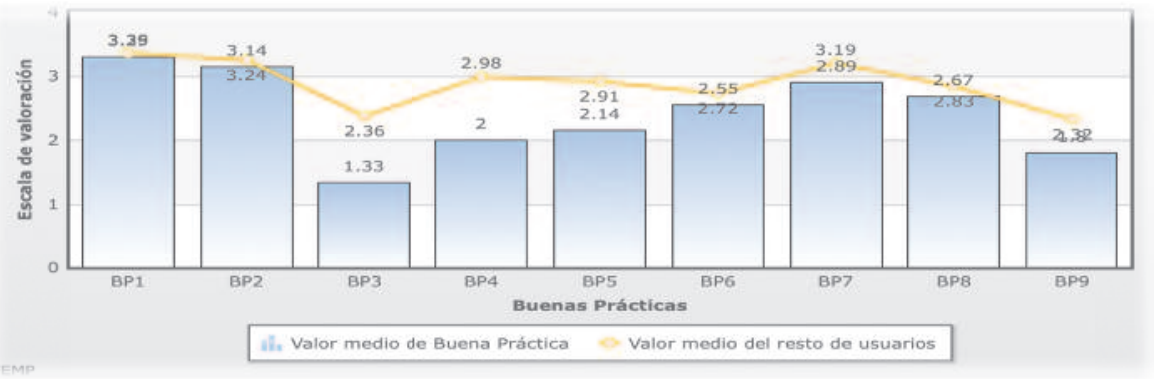

\section{Fortalezas del servicio.}

Los objetivos y las actuaciones del servicio se rigen por el interés superior del menor y las necesidades de desarrollo de la infancia y la adolescencia, enfatizan el deber tanto de la madre como del padre u otras figuras parentales de educar positivamente a los hijos e hijas. Se facilita que los usuarios conozcan sus derechos y deberes. Se fomentan relaciones basadas en el respeto a la diversidad familiar, cultural, socioeconómica y de género en las familias. Las acciones van encaminadas a que las figuras parentales eduquen positivamente a los hijos e hijas mediante unas relaciones afectivas sanas, seguras y estables en la familia. Se fomentan el establecimiento de unas buenas relaciones padres-hijos basadas en la comunicación, establecimiento de límites, la supervisión, acepta- 


\section{EXPERIENCIA DE LA APLICACION DEL PROTOCOLO DE EVALUACIÓN DE BUENAS PRÁCTICAS EN PARENTALIDAD POSITIVA EN EL PROGRAMA DE ACOGIMIENTO RESIDENCIAL DE MENORES GESTIONADO POR LA ENTIDAD MENSAJEROS DE LA PAZ- CANARIAS}

ción mutua, apoyo, solución de problemas. De igual forma se informa a las figuras parentales y en particular a aquellos con carencias socioculturales de los recursos de apoyo de todo tipo (vg., apoyo psico-educativo) existentes en la comunidad.

Los y las profesionales del servicio tienen capacidad para trabajar colaborativamente con las familias. Existen protocolos de derivación para cuando se detectan dificultades o necesidades de las familias que deban ser atendidas por otros servicios más especializados.

\section{Recomendaciones para el servicio.}

Desde el servicio se debería organizar actividades formativas para que los y las profesionales y la comunidad conozcan los derechos del niño y del adolescente, así como organizar acciones formativas para profesionales sobre el enfoque de la parentalidad positiva. El servicio debería disponer de guías y/o folletos sobre los programas y acciones de parentalidad positiva destinados a todas las familias. Destinar tiempo para establecer espacios de reflexión para promover la innovación y las buenas prácticas. Evaluar el resultado de los modelos o prácticas profesionales en función de los objetivos planteados. Se detecta la necesidad de valorar y fomentar la colaboración con las universidades para el diseño, implementación y evaluación de programas y difundir los resultados de las colaboraciones con la universidad entre los y las profesionales y, cuando sea conveniente, en la comunidad.

PARTE 2. Buenas prácticas con el trabajo profesional con las familias desde el enfoque de la parentalidad positiva.

Figura 2. Resultados del protocolo de Buenas Prácticas en parentalidad positiva. FEMP.

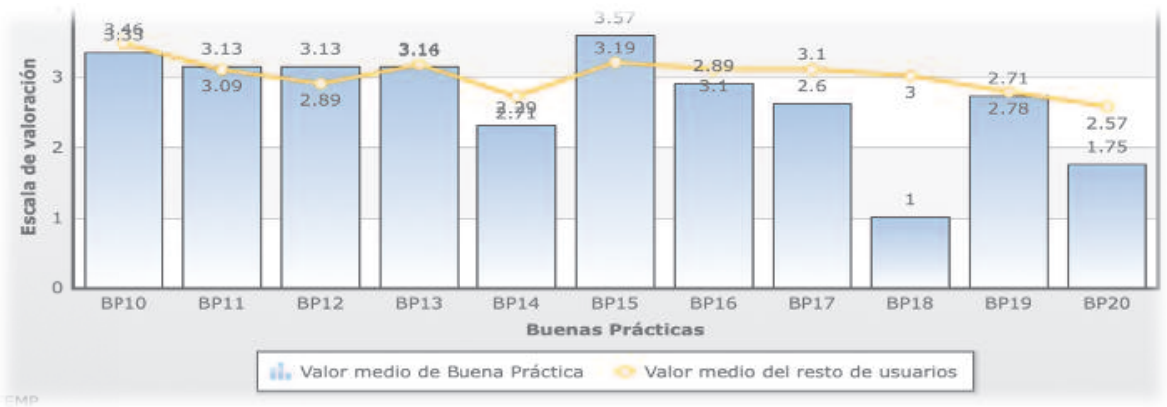

Fortalezas del servicio.

En las actuaciones del servicio se anima y promueve la colaboración de las familias. Los y las profesionales están atentos a reconocer sus propios prejuicios sobre las familias y a evitar cualquier actitud que interfiera con el trato con las familias. En las actuaciones del servicio se da a conocer a la familia los recursos existentes en la comunidad que pueden cubrir sus necesidades de apoyo social. Se mantiene una visión positiva y detallada del funcionamiento familiar, evitando las valoraciones globales y descalificadoras. En las actuaciones del servicio se analizan/evalúan las actitudes y expectativas de los padres/madres o figuras parentales acerca del desarrollo y la educación de los hijos e hijas, así como la capacidad de los padres/madres o figuras parentales para organizar y estructurar de forma adecuada la vida cotidiana de los hijos/as y de la familia.

En el trabajo con las familias se comprueba si los padres y las madres se sienten capaces de 
realizar su labor educadora y se analiza/evalúa la adaptación del niño, niña y adolescente en su contexto familiar y a los distintos contextos de desarrollo en los que participan fuera de la familia (escuela, compañeros, deporte, salud, ocio, etc.). Se favorece que éstas reconozcan sus logros y experiencias positivas anteriores para promover su confianza en los retos que se proponen. Se les pregunta a las familias por las buenas cualidades que tienen sus hijos e hijas (vg. personalidad, comportamiento, habilidades, aficiones) y se les hace reflexionar sobre su proyecto de vida: metas, aspiraciones para ellos y para sus hijos e hijas y cómo la intervención puede ponerles en camino de conseguirlo. Desde el servicio se fomenta la buena relación de las familias con otros contextos importantes para el desarrollo y la educación de los hijos e hijas (escuela, barrio, amigos, ocio). Se describen las cualidades positivas de los diferentes miembros de la familia. Se lleva a cabo una supervisión de la intervención compartida con los y las profesionales que intervienen. Se utilizan actividades de atención individual estructuradas y encaminadas a que la familia mejore las pautas relacionadas con la salud, el cuidado y educación de los hijos, el apoyo escolar y el ocio familiar, adaptadas a la familia. Se utiliza la modalidad grupal en el servicio para mejorar las competencias y resiliencia de los niños y adolescentes, como por ejemplo a través de las asambleas en los hogares, en estas intervenciones se plantean objetivos de cambio que fomenten el desarrollo positivo, relaciones sanas entre iguales, su participación en la comunidad y el ocio adecuado en el grupo de iguales.

Para la evaluación familiar se utilizan distintas fuentes de información: entrevista, visitas domiciliarias, cuestionarios, observación, etc. para el análisis o evaluación de la/s situación/es familiar/es.

\section{Recomendaciones para el servicio.}

En el trabajo con las familias se debería comprobar si los padres y las madres están satisfechos con el rol parental. En las actuaciones del servicio se debería usar un protocolo de evaluación familiar consensuado, teóricamente fundamentado y compartido por todos los y las profesionales responsables de la atención a las familias. En las actuaciones del servicio se deberían utilizar instrumentos de evaluación validados y baremados para la población destinataria en la valoración de algunos de los aspectos del ejercicio de la parentalidad. Se deberían utilizar instrumentos de evaluación validados y baremados para la población destinataria en la valoración del impacto de la parentalidad sobre el bienestar de los hijos/as. Se debería contar con un informe de evaluación final que permita integrar la información recabada por distintos profesionales en el trabajo con las familias. En las actuaciones del servicio se debería preguntar a las familias lo que esperan del profesional y de la intervención. Se debería utilizar la modalidad grupal en los planes de intervención para promover, entre otros aspectos, la parentalidad positiva. Además, se debería capacitar a la comunidad para el desarrollo de acciones preventivas y de promoción para los padres/madres y para los niños y adolescentes, así como promover programas que potencien la relación entre familia, escuela, salud y comunidad. Desde el servicio se deberían diseñar estrategias de trabajo para fortalecer tanto la participación ciudadana activa como la articulación de la comunidad (vg., asociaciones, voluntariado). 


\section{EXPERIENCIA DE LA APLICACION DEL PROTOCOLO DE EVALUACIÓN DE BUENAS PRÁCTICAS EN PARENTALIDAD POSITIVA EN EL PROGRAMA DE ACOGIMIENTO RESIDENCIAL DE MENORES GESTIONADO POR LA ENTIDAD MENSAJEROS DE LA PAZ- CANARIAS}

\section{PARTE 3. Programas reunificación familiar.}

Figura 3. Resultados del protocolo de Buenas Prácticas en parentalidad positiva. FEMP.

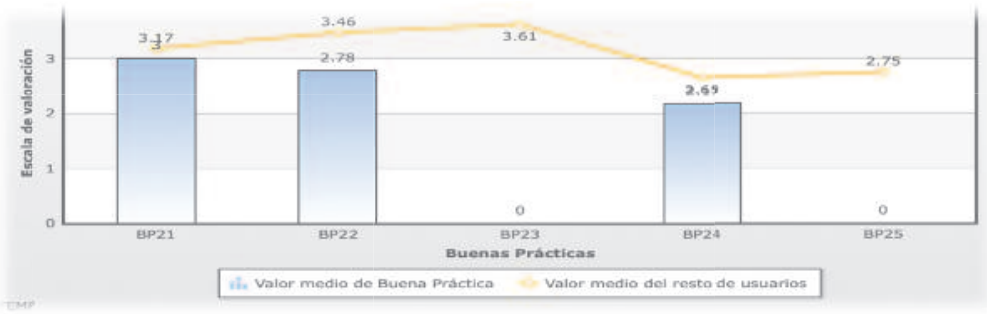

\section{Fortalezas del servicio.}

El propio servicio aporta contenidos a tratar en los programas basándose en su propio sistema de recogida de información sobre las familias. Durante el desarrollo de los programas se registra el índice de asistencia a las sesiones de cada participante y las razones de las posibles ausencias.

\section{Recomendaciones para el servicio.}

Se debería incluir grupo de control y grupo de intervención en el diseño de evaluación de los programas. Así como incluir procedimientos y recursos para valorar el grado de eficacia de la gestión institucional en la aplicación de los programas.

Se debería contar con una evaluación externa y rigurosa de los programas, además de las propias evaluaciones que desarrolla el servicio. Se debería disponer de procedimientos para valorar el grado de satisfacción de las personas participantes en los programas.

\section{PLAN DE MEJORA}

Con el objetivo de alcanzar mejoras en el servicio de atención a las familias por parte de la entidad se han puesto en marcha las siguientes acciones tras la aplicación del protocolo priorizando por necesidad detectada. Se han establecido objetivos a corto plazo y a largo plazo ajustándose a la viabilidad de la puesta en marcha desde la entidad con metas temporalizadas que puedan ser realistas y alcanzables.

\section{OBJETIVOS A CORTO PLAZO.}

BP3. ESTABLECER CANALES DE SENSIBILIZACIÓN E INFORMACIÓN PARA FACILITAR EL ACCESO UNIVERSAL AL SERVICIO.

El servicio debería disponer de guías y/o folletos sobre los programas y acciones de parentalidad positiva destinados a todas las familias. (en este caso debido a la particularidad del servicio no va dirigido a toda la población en general sino a las familias con las que se interviene de manera directa o indirecta desde el servicio).

ACCIÓN DE LA ENTIDAD: Elaborar folletos/guías informativas donde se expliquen a las familias el que consiste le programa de acogimiento residencial de menores, su funcionamiento, el proceso, y los objetivos a alcanzar desde el enfoque de la parentalidad positiva que sirva como soporte de información, aunque requiera una explicación por parte del o la profesional adecuada al usuario/a de acuerdo con su comprensión, lenguaje claro y sencillo.

BP5. EMPLEAR MODELOS Y PRÁCTICAS PROFESIONALES BASADAS EN EL CONSENSO Y LA EVIDENCIA CIENTÍFICA. El servicio debería destinar tiempo para establecer espacios de reflexión para promover la innovación y las buenas prácticas. 
ACCIÓN DE LA ENTIDAD: De manera trimestral se crearán espacios de trabajo con los profesionales responsables del programa con el fin de intercambiar impresiones, instrumentos validados, líneas de trabajo desde el enfoque de la parentalidad positiva.

BP14. LLEVAR A CABO UNA EVALUACIÓN RIGUROSA DE LAS CONDICIONES EN LAS QUE SE EJERCE LA PARENTALIDAD EN LAS FAMILIAS. En las actuaciones del servicio se debería usar un protocolo de evaluación familiar consensuado, teóricamente fundamentado y compartido por todos los y las profesionales responsables de la atención a las familias.

ACCIÓN DE LA ENTIDAD: Utilización de instrumentos validados y baremados sobre las dinámicas y funcionamiento familiar.

\section{OBJETIVOS A LARGO PLAZO}

BP18.REALIZAR ACTIVIDADES GRUPALES ESTRUCTURADAS PARA LA PROMOCIÓN DE LA PARENTALIDAD. Se debería utilizar la modalidad grupal en los planes de intervención para promover, entre otros aspectos, la parentalidad positiva.

ACCIÓN DE LA ENTIDAD: Emprender acciones para poner en marcha programas grupales basados en evidencias destinados a grupos de padres, familiares donde se trabaje el pronóstico de reunificación familiar o acogimiento en familia extensa de los/as menores.

\section{CONCLUSIONES}

Tras la puesta en marcha del plan de mejora en la entidad se han obtenido resultados positivos. Este plan de mejora se encuentra en fase de implementación, no obstante, estos cambios ya están produciendo mejoras tanto en las respuestas a las intervenciones de las propias familias como en el trabajo diario de los profesionales poniendo en marcha una metodología de trabajo rigurosa, consensuada y conjunta por parte de todo el equipo. Las valoraciones obtenidas por los profesionales del servicio se traducen por el momento en:

Las mejoras en el servicio aportan rigor y profesionalidad a la labor de análisis del trabajo familiar y educativo con los/as menores.

Permite concretar con mayor exactitud aquellos aspectos susceptibles de mejoras o intervenciones a distintos niveles, conociendo los factores de protección y discriminando lo que no sea relevante en el momento de la intervención para la mejora de la situación de una forma objetiva.

La aplicación de programas basados en evidencias, así como la utilización de instrumentos basados en la evidencia científica para el análisis familiar permite identificar por parte de todos los profesionales un mayor rango de actuación, visibilizando aspectos que podrían no haber sido percibidos

Se aporta transparencia en la intervención con las familias mejorando la relación de ayuda al comprender las familias el papel que desempeña el profesional en su quehacer diario.

\section{REFERENCIAS BIBLIOGRÁFICAS}

Mensajeros de la Paz -Canarias (2018). Programa de Centro. Anexos: Programas de intervención familiar en acogimiento residencial. Santa Cruz de Tenerife.

Rodrigo, M.J., Amoros, P., Arranz, E., Hidalgo, M.V., Maiquez, M.L., Martin, J.C., Martinez, R.A. y Ochaita, E. (2015). Guia de Buenas Practicas en Parentalidad Positiva. Un recurso para apoyar la practica profesional con familias. Madrid: Federacion Espanola de Municipios y Provincias (FEMP).

Rodrigo, M.J., Maiquez, M.L. y Martin, J.C. (2010b). La educacion parental como recurso psicoeducativo para promover la parentalidad positiva. Madrid: Federacion Espanola de Municipios y Provincias (FEMP). 
EXPERIENCIA DE LA APLICACION DEL PROTOCOLO DE EVALUACIÓN DE BUENAS PRÁCTICAS EN PARENTALIDAd

POSITIVA EN EL PROGRAMA DE ACOGIMIENTO RESIDENCIAL DE MENORES GESTIONADO POR LA ENTIDAD

MENSAJEROS DE LA PAZ- CANARIAS

Rodrigo, M.J., Maiquez, M.L. y Martin, J.C. (2011). Buenas practicas profesionales para el apoyo de la parentalidad positiva. Madrid: Federacion Espanola de Municipios y Provincias (FEMP). 